\title{
The Harder the Heart, the Harder It Breaks: A Case of Complete Atrioventricular Block Secondary to Tertiary Hyperparathyroidism
}

\author{
Ramanakumar Anam ${ }^{1}$, Mauricio Tellez ${ }^{1}$, Matthew Lozier ${ }^{2}$, Ameen Waheed ${ }^{3}$, Jose E. Collado ${ }^{4,5}$ \\ 1. Internal Medicine, University of Miami at Holy Cross Hospital, Fort Lauderdale, USA 2. Cardiology, Mount Sinai \\ Medical Center, Miami, USA 3. Nephrology, University of Miami at Holy Cross Hospital, Fort Lauderdale, USA 4. \\ Cardiology, University of Miami at Holy Cross Hospital, Fort Lauderdale, USA 5. Cardiology, Jim Moran Heart \& \\ Vascular Center at Holy Cross Hospital, Fort Lauderdale, USA
}

Corresponding author: Ramanakumar Anam,dr.ramankumar@gmail.com

\begin{abstract}
The appearance of first-degree atrioventricular block and mitral annulus calcification in an end-stage renal failure patient with elevated parathyroid hormone levels should raise the suspicion of metastatic cardiac calcification. Measures should be taken to normalize the parathyroid hormone, calcium, and phosphorus levels to limit the progression of atrioventricular block. Exploration or removal of parathyroid glands should be considered if heart block worsens.
\end{abstract}

Review began 01/31/2021 Review ended 02/06/2021 Published 02/11/2021

\section{() Copyright 2021}

Anam et al. This is an open access article distributed under the terms of the Creative Commons Attribution License CC-BY 4.0., which permits unrestricted use, distribution, and reproduction in any medium, provided the original author and source are credited.
Categories: Cardiology, Endocrinology/Diabetes/Metabolism, Nephrology

Keywords: mitral annular calcification, tertiary hyperparathyroidism, atrioventricular conduction block, hemodialysis, end stage renal disease

\section{Introduction}

Since the End-Stage Renal Disease (ESRD) Program initiation in 1972, medical complications, including metastatic calcifications, have been rising [1,2]. Gradual deposition of calcium within the cardiac conduction system may predispose these patients to an increased incidence of arrhythmias, heart failure, and mortality.

We present the case of a 68-year-old male previously diagnosed with ESRD on hemodialysis, who developed a third-degree atrioventricular (AV) block attributed to calcification of the mitral valve annulus. While this patient required placement of a permanent pacemaker, this report's objective was to retrospectively assess findings that may have predicted and allowed the ability to prevent this outcome.

\section{Case Presentation}

A 68-year-old male previously diagnosed with ESRD on hemodialysis presented to the ED due to lightheadedness and exertional dyspnea for the past several weeks. These symptoms were associated with multiple syncopal events that affected his quality of life.

His past medical history was significant for hypertension and type 2 diabetes, and he was a social drinker but non-smoker with no adverse family history. His home medications included calcium acetate $667 \mathrm{mg}$ thrice daily, cinacalcet $60 \mathrm{mg}$ once daily, gabapentin $300 \mathrm{mg}$, rosuvastatin $40 \mathrm{mg}$, and thiamine $200 \mathrm{mg}$.

In the $\mathrm{ED}$, his vital signs were stable, and he appeared to be in no distress. A cardiac examination was significant for $2 \mathrm{~cm}$ jugular venous distention and a mid-systolic click in the mitral area. While initial laboratory values, including calcium (Ca, corrected $9.6 \mathrm{mg} / \mathrm{dL}$ ) and phosphorus (P, $3.7 \mathrm{mg} / \mathrm{dL}$ ), were mostly unremarkable, parathyroid hormone (PTH) level was elevated at $737 \mathrm{pg} / \mathrm{mL}$. An electrocardiogram (EKG) in the ED revealed a first-degree AV block with a PR interval of $300 \mathrm{~ms}$; however, telemetry was later notable for complete heart block (Figure 1). Electrophysiology placed an emergent temporary transvenous pacemaker, and he was admitted to the floor. The following day he received a dual-chamber permanent pacemaker. Subsequent transthoracic echocardiogram demonstrated severe mitral annular calcification (MAC; four videos in the Appendix), which was thought to be the underlying etiology for developing the cardiac conduction disorder. 


\section{Cureus}
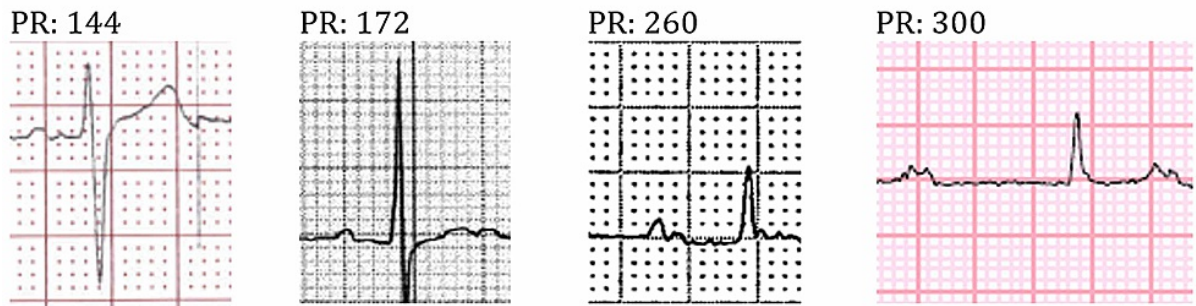

Complete heart block:

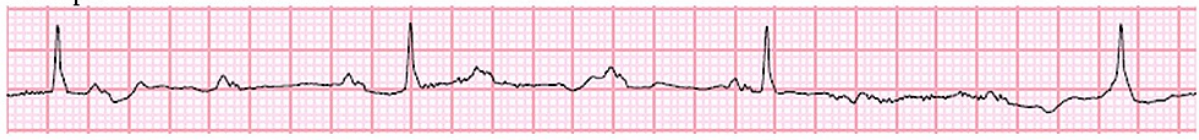

FIGURE 1: EKG illustrating sequential worsening of the atrioventricular block

EKG: electrocardiogram

A retrospective chart review of prior serial testing demonstrated a progressively increasing PR interval and PTH level over the last several years. These findings appear to be directly correlated with worsening mitral annulus calcification (Table 1, Figure 2). Chest CT comparison between 2013 (without contrast) and 2019 (with contrast) shows worsening of MAC calcification from 2013 to 2019 (figure in the Appendix).

\begin{tabular}{|c|c|c|c|c|c|}
\hline MM/YY & Corrected Ca (mg/dL) & Ca $\times$ P & PR interval (ms) & Echo & PTH (pg/mL) \\
\hline 12/12 & 10.3 & 65 & 144 & Normal study & 447 \\
\hline 09/14 & 11.1 & 71 & 172 & Mild MAC & 537 \\
\hline 02/16 & 9.8 & 73 & 260 & Moderate MAC & 629 \\
\hline 11/19 & 10 & 39 & 300 & Severe MAC & 737 \\
\hline
\end{tabular}

\section{TABLE 1: Serial laboratory values, EKG, and echo findings}

\section{Ca: calcium; Ca x P: calcium phosphate product; PTH: parathyroid hormone; EKG: electrocardiogram}

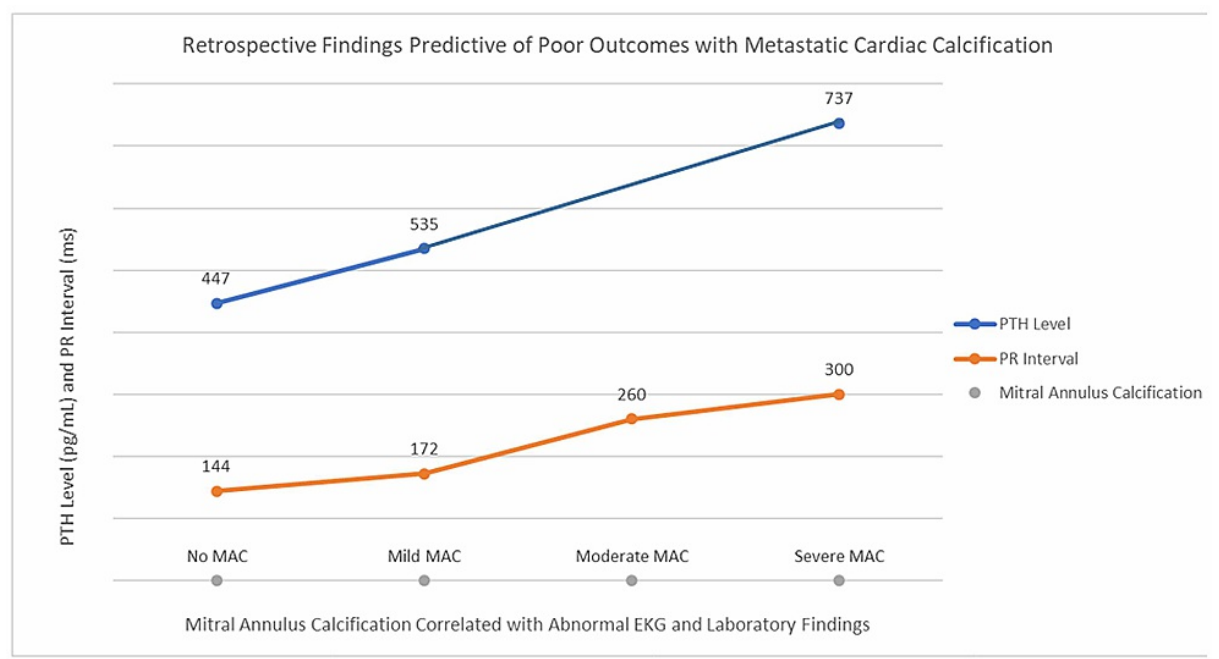

FIGURE 2: Shows worsening of PR interval and MAC as the serum PTH level increases

MAC: mitral annulus calcification; PTH: parathyroid hormone 


\section{Discussion}

Despite the prompt use of vitamin D metabolites in ESRD patients on hemodialysis, calcium and phosphorus disorders are common. These metabolic abnormalities can result in calcium deposition in normal healthy soft tissues, known as metastatic calcification. Metastatic calcification is an important etiology of cardiac conduction disorders for this subpopulation of patients.

Varma et al. demonstrated MAC to occur in 10-50\% of ESRD patients [3]. Furthermore, Forman et al. demonstrated that MAC occurs more frequently in those receiving dialysis for an extended period [4]. This is notable for the current report as MAC is associated with a high frequency of conduction defects. Nair et al. found MAC in $87 \%$ of patients with symptomatic bradyarrhythmias [5]. While the association and mechanism between MAC and conduction disease have not been fully elucidated, the current hypothesis supports the notion that it may be partially due to the direct extension of calcium deposits in the AV node region and His bundle [6-8].

Proposed pathologic mechanisms in literature behind the metastatic calcifications in ESRD patients on hemodialysis include hyperparathyroidism, $\mathrm{Ca}$ and $\mathrm{P}$ products more than 72 influenced by dialysate calcium concentration, and vitamin D3 [9,10]. Few preliminary studies have shown that myocardial hypertrophy due to the elevated PTH and Ca have a similar effect. However, Barletta et al. hypothesized that PTH influences calcium entry into myocardial tissues independent of serum calcium level [11].

The salient features were metastatic calcification in the myocardium and the cardiac conduction system in a patient on cinacalcet and calcium acetate. Our patient was diagnosed with ESRD and secondary hyperparathyroidism about 10 years before this hospitalization and has been on dialysis since then. Serial echocardiogram obtained approximately every two to three years has shown a gradual increase in the MAC over the past 10 years. Serial EKG was also reviewed for the same duration and revealed a progressive PR interval prolongation that started after moderate MAC, which was seen on the transthoracic echocardiogram in 2017. Knowing that MAC is associated with an elevated calcium-phosphorus (Ca x P) product, hypercalcemia, and hyperphosphatemia - we reviewed his labs for the past 10 years that were available on the electronic health record. Records support a diagnosis of tertiary hyperparathyroidism with elevated PTH, elevated phosphorus even though he did not have overt hypercalcemia (Table 1). He was treated with calcimimetic drugs from early in the course of the disease process.

We conclude that the elevated PTH could be the driving force of calcium deposition in the mitral annulus in the absence of high calcium and elevated Ca x P. Prior case reports have demonstrated the reversal of AV block after parathyroidectomy $[12,13]$. However, there is a lack of evidence supporting early parathyroidectomy after first-degree AV block's appearance in long-term hemodialysis patients with secondary/tertiary hyperparathyroidism. Measures should be taken to optimize serum levels of PTH, calcium, and phosphate. Exploration and removal of parathyroid glands should be considered, when feasible, to prevent further progression of AV block.

\section{Conclusions}

Long-term hemodialysis patients are predisposed to the development of cardiac conduction disorders. The appearance of a first-degree atrioventricular block in the setting of an elevated parathyroid hormone level and calcification of the mitral valve annulus may warrant serial EKG testing to assess for more pronounced deviations from the normal cardiac conduction. With the recognition of these findings, early consideration of parathyroidectomy should occur to prevent the worsening of the AV block.

\section{Appendices}




\section{Cureus}

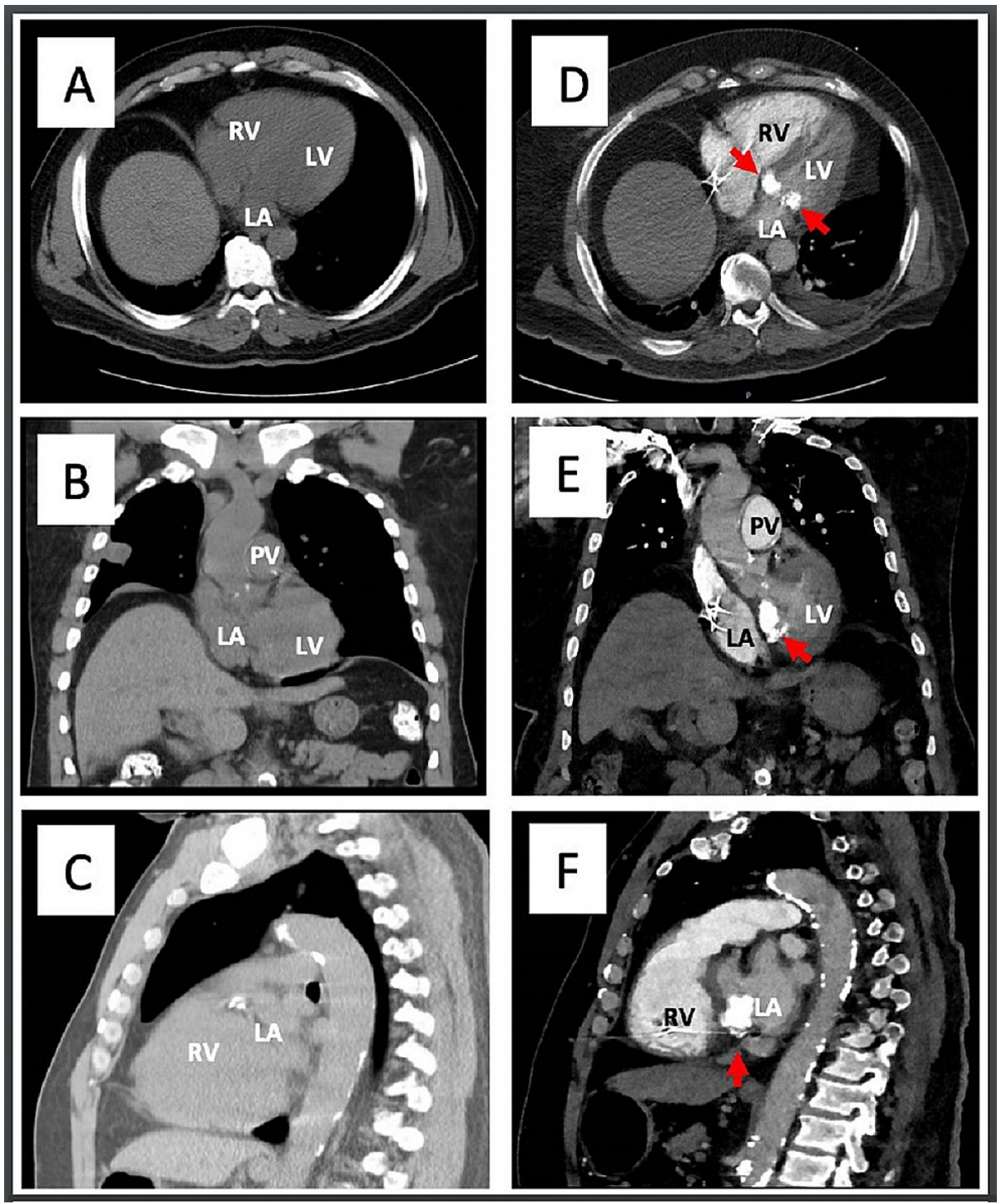

FIGURE 3: CT chest

Chest CT comparison between 2013 (without contrast) and 2019 (with contrast). A. Axial view 2013; B.

coronal view 2013; C. sagittal view 2013 D. axial view 2019; E. coronal view 2019; F. sagittal view 2019. Red arrow shows a heavily calcified mitral valve.

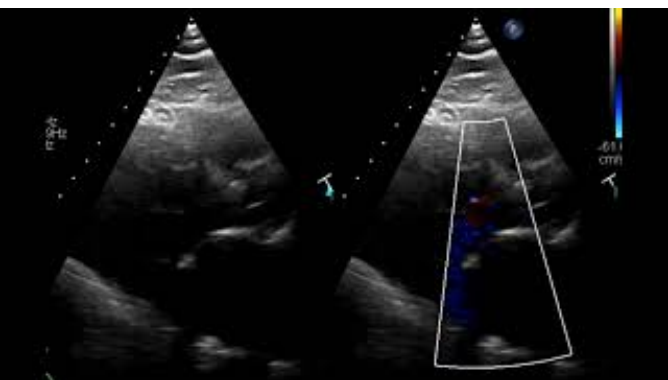

VIDEO 1: Parasternal long-axis view

View video here: https://youtu.be/KQrmZDFise0 


\section{Cureus}

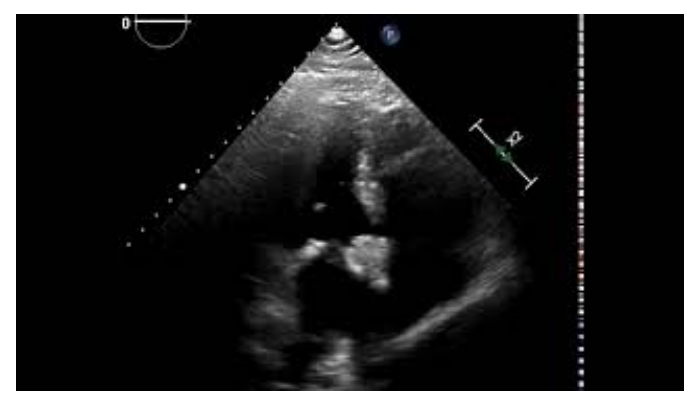

VIDEO 2: Apical three-chamber view

View video here: https://youtu.be/VFXGf8nXSGc

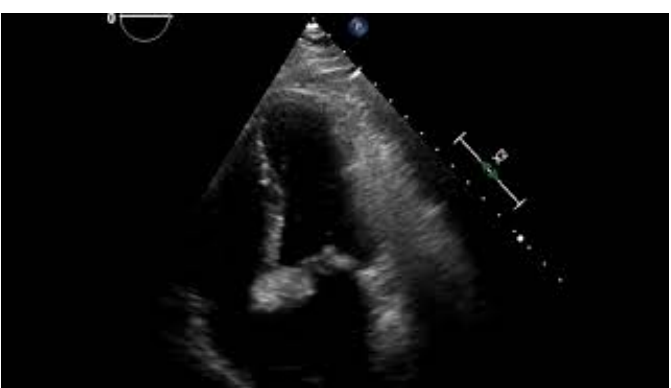

VIDEO 3: Apical four-chamber view

View video here: https://youtu.be/I9td4ZaSkBI

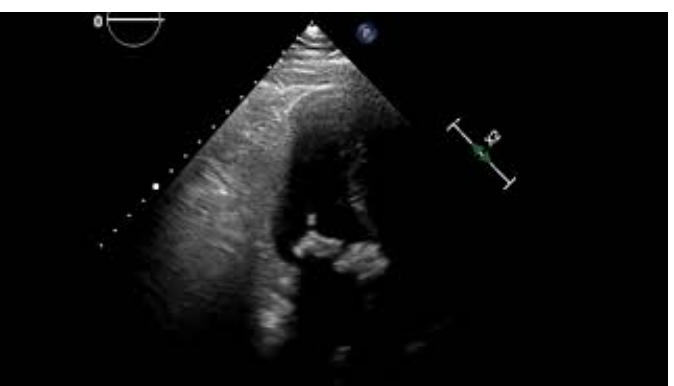

VIDEO 4: Apical two-chamber view

View video here: https://youtu.be/EBR_3Pcl9el

\section{Additional Information}

\section{Disclosures}

Human subjects: Consent was obtained or waived by all participants in this study. Conflicts of interest: In compliance with the ICMJE uniform disclosure form, all authors declare the following: Payment/services info: All authors have declared that no financial support was received from any organization for the submitted work. Financial relationships: All authors have declared that they have no financial relationships at present or within the previous three years with any organizations that might have an interest in the submitted work. Other relationships: All authors have declared that there are no other relationships or activities that could appear to have influenced the submitted work.

\section{References}

1. Leier CV, Boudoulas H: Renal disorders and heart disease. Heart Disease. A Textbook of Cardiovascular Medicine. Braunwald E (ed): W.B. Saunders Company, Philadelphia, USA; 1997. 1914-1938.

2. Terman DS, Alfrey AC, Hammond WS, Donndelinger T, Ogden DA, Holmes JH: Cardiac calcification in uremia. A clinical, biochemical, and pathologic study. Am J Med. 1971, 50:744-755. 10.1016/00029343(71)90182-3

3. Varma R, Aronow WS, McClung JA, Garrick R, Vistainer PF, Weiss MB, Belkin RN: Prevalence of valve calcium and association of valve calcium with coronary artery disease, atherosclerotic vascular disease, and all-cause mortality in 137 patients undergoing hemodialysis for chronic renal failure. Am J Cardiol. 2005, 


\section{Cureus}

95:742-743. 10.1016/j.amjcard.2004.11.025

4. Forman MB, Virmani R, Robertson RM, Stone WJ: Mitral annular calcification in chronic renal failure . Chest. 1984, 85:367-371. 10.1378/chest.85.3.367

5. Nair CK, Sketch MH, Desai R, Mohiuddin SM, Runco V: High prevalence of symptomatic bradyarrhythmias due to atrioventricular node-fascicular and sinus node-atrial disease in patients with mitral anular calcification. Am Heart J. 1982, 103:226-229. 10.1016/0002-8703(82)90496-3

6. Fulkerson PK, Beaver BM, Auseon JC, Graber HL: Calcification of the mitral annulus: etiology, clinical associations, complications and therapy. Am J Med. 1979, 66:967-977. 10.1016/0002-9343(79)90452-2

7. Nestico PF, Depace NL, Morganroth J, Kotler MN, Ross J: Mitral annular calcification: clinical, pathophysiology, and echocardiographic review. Am Heart J. 1984, 107:989-996. 10.1016/00028703(84)90840-8

8. Davies MI, Anderson RH: The pathology of the conduction system. The Pathology of the Heart. Pomerance A, Davies MJ (ed): Blackwell Scientific, London, UK; 1975. 367-412.

9. McCarthy JT, El-Azhary RA, Patzelt MT, et al.: Survival, risk factors, and effect of treatment in 101 patients with calciphylaxis. Mayo Clin Proc. 2016, 91:1384-1394. 10.1016/j.mayocp.2016.06.025

10. Arora KK, Lacy JP, Schacht RA, Martin DG, Gutch CF: Calcific cardiomyopathy in advanced renal failure . Arch Intern Med. 1975, 135:603-605. 10.1001/archinte.1975.00330040115019

11. Barletta G, De Feo ML, Del Bene R, et al.: Cardiovascular effects of parathyroid hormone: a study in healthy subjects and normotensive patients with mild primary hyperparathyroidism. J Clin Endocrinol Metab. 2000, 85:1815-1821. 10.1210/jcem.85.5.6514

12. Dreher W, Shelp W: Atrioventricular block in a long-term dialysis patient. Reversal after parathyroidectomy . JAMA. 1975, 234:954-955. 10.1001/jama.1975.03260220058021

13. Fujimoto S, Hisanaga S, Yamatomo Y, Tanaka K, Hayashi T, Sumiyoshi A: Tertiary hyperparathyroidism associated with metastatic cardiac calcification in a haemodialyzed patient. Int Urol Nephrol. 1991, 23:285292. 10.1007/bf02550426 